Pacific Journal of Mathematic 


\title{
UNIMODULAR APPROXIMATION IN FUNCTION ALGEBRAS
}

\author{
JOHN N. MC DONALD
}

\begin{abstract}
Let $A$ be a function algebra on the compact Hausdorff space $X$. The main result of this paper gives necessary and sufficient conditions for the set of quotients of inner functions in $A$ to be dense in the set of continuous unimodular functions on $X$. A theorem of Douglas and Rudin concerning quotients of Blaschke products is derived. The main result is also applied in the context of the theory of compact abelian groups.
\end{abstract}

The prototype for the main result of this paper is the following theorem due to Douglas and Rudin [3].

THEOREM 1. Suppose that $u$ is a measurable function on the unit circle such that $\left|u\left(e^{i t}\right)\right|=1$ a.e. Then $u$ can be approximated arbitrarily closely in the essential-sup norm by quotients of Blaschke products.

We will derive Theorem 1 from a general result concerning function algebras. Our main result will also be applied to certain algebras of functions on compact abelian groups.

Preliminaries. Consider a sub-algebra $A$ of the algebra $C(X)$ of continuous complex-valued functions on the compact Hausdorff space $X$. We will assume that $A$ contains the constant functions and is closed with respect to the sup-norm $\|\cdots\|$. We will call a function $g \in A$ inner if $|g| \equiv 1$. Let $I(A)$ denote the set of inner functions and let $Q(A)$ denote the set of quotients of inner functions. Of course the set $U(X)=I(C(X))$ is simply the collection of continuous unimodular functions on $X . U(X)$ is a group under pointwise multiplication. $U(X)$ has an important subgroup, namely the group $\log U(X)$ of members of $U(X)$ having continuous logarithms. We will indicate the natural quotient map from $U(X)$ to $U(X) / \log U(X)$ by $\pi_{X}$.

In the case where $X$ is the $n$-dimensional torus $T^{n}$ and $A$ is the polydisk algebra $A\left(T^{n}\right)$, i.e., the closed algebra generated by the coordinate projections from $T^{n}$ onto $T^{1}$, there is an abundance of inner functions. It is known that a function $f \in A\left(T^{n}\right)$ is inner if and only if it is of the form

$$
f(z)=M(z) \hat{Q}(z) / Q(z), \quad z=\left(z_{1}, z_{2}, \ldots, z_{n}\right),
$$


where $M$ is a monomial, $Q(z)$ is a polynomial of degree $d(j)$ in $z_{j}$ for $j=1,2, \ldots, n$ which does not vanish anywhere on the closed unit polydisk, and $\hat{Q}(z)$ is the unique polynomial satisfying

$$
\hat{Q}(z)=z_{1}^{d(1)} z_{2}^{d(2)} \cdots z_{n}^{d(n)} \overline{Q(z)}
$$

for every $z \in T^{n}$. See [6, p. 112]. Note in particular that every $f \in I\left(A\left(T^{n}\right)\right)$ is a rational function.

Our reason for discussing inner functions in the polydisk algebra is that they are related to the general case via the following easily proved:

Proposition 2. Let $A$ be the general function algebra as described above. Let $f_{1}, f_{2}, \ldots, f_{n} \in I(A)$ and $F \in A\left(T^{n}\right)$, then $F\left(f_{1}, f_{2}, \ldots, f_{n}\right) \in A$. In particular, if $F \in I\left(A\left(T^{n}\right)\right)$, then $F\left(f_{1}, f_{2}, \ldots, f_{n}\right) \in I(A)$.

\section{Main Result.}

THEOREM 3. Let $A$ be as described above. $Q(A)$ is dense in $U(X)$ if and only if the following conditions hold.

(i) $\pi_{X}(Q(A))=U(X) / \log U(X)$

(ii) $I(A)$ separates the points of $X$.

Proof. Suppose that $Q(A)$ is dense in $U(X)$. Let $u \in U(X)$. Since $\log U(X)$ is an open subgroup of $U(X)$ containing the constant function 1 , it follows that there exists a $q \in Q(A)$ such that $\bar{q} u \in \log U(X)$. Thus, we have $\pi_{X}(u)=\pi_{X}(q)$. It follows that (i) is satisfied. Next, suppose $a, b \in X$ with $a \neq b$. It's easy to find a function $u_{0} \in U(X)$ such that $u_{0}(a) \neq u_{0}(b)$. If $g(a)$ were equal to $g(b)$ for every $g$ in $I(A)$, then it would follow that $q(a)=q(b)$ for every $q \in Q(A)$. But by choosing a $q_{0} \in Q(A)$ sufficiently close to $u_{0}$ we can obtain $q_{0}(a) \neq q_{0}(b)$. This contradiction shows that we must have $g_{0}(a) \neq g_{0}(b)$ for some $g_{0} \in I(A)$.

Now we will assume that (i) and (ii) hold. To show that $Q(A)$ is dense in $U(X)$ we observe first that the uniform closure $\overline{Q(A)}$ of $Q(A)$ is a subgroup of $U(X)$. Next we note that, because of (i), it suffices to show that $\log U(X)$ is contained in $\overline{Q(A)}$. A function $u$ in $U(X)$ belongs to $\log U(X)$ if and only if it has the form

$$
u=e^{i r}
$$

where $r$ is a continuous real valued function on $X$. Since

$$
u=\left(e^{i r / n}\right)^{n}
$$

for $n=1,2, \ldots$, it follows that each member of $\log U(X)$ can be written as an integer power of a function in $U(X)$ which is arbitrarily close to the 
constant function 1 . Thus, to show $\log U(X) \subseteq \overline{Q(A)}$ it is enough to show that $\overline{Q(A)}$ contains all functions of the form

$$
u=v^{2}
$$

where $v \in U(X)$ and $\operatorname{Re} v>1 / 2$.

Let $u$ and $v$ be as in (1) and let $\varepsilon \in\left(0, \frac{1}{4}\right)$. By (ii) and the StoneWeierstrass theorem we can find inner functions $g_{1}, g_{2}, \ldots, g_{n}, h_{1}$, $h_{2}, \ldots, h_{n}$ and constants $c_{1}, c_{2}, \ldots, c_{n}$ such that

$$
\left\|v-\sum_{j=1}^{n} c_{j} \bar{g}_{j} h_{j}\right\|<\varepsilon .
$$

Clearly, (2) can be re-written in the form

$$
\left\|v-\bar{g} \sum_{j=1}^{n} c_{j} f_{j}\right\|<\varepsilon,
$$

where $g=\prod_{j=1}^{n} g_{j}$ and $f_{1}, f_{2}, \ldots, f_{n} \in I(A)$. It follows easily from (3) that

$$
\operatorname{Re} \bar{g} \sum_{j=1}^{n} c_{j} f_{j}>\frac{1}{4}
$$

Since $v=1 / v$, it follows again from (3) that

$$
\left\|v-\bar{g} / \sum_{j=1}^{n} \bar{c}_{j} \bar{f}_{j}\right\|<4 \varepsilon .
$$

Thus, we have

$$
\left\|u-\bar{g}^{2} \sum_{j=1}^{n} c_{j} f_{n} / \sum_{j=1}^{n} \bar{c}_{j} \tilde{f}_{j}\right\|<8 \varepsilon .
$$

We now define a mapping $\Psi: X \rightarrow T^{n+1}$ via

$$
\Psi(x)=\left(g(x), f_{1}(x), \ldots, f_{n}(x)\right) .
$$

Consider the function as defined on $\Psi(X)$ by

$$
r(z)=r\left(z_{0}, z_{1}, \ldots, z_{n}\right)=\bar{z}_{0} \sum_{j=1}^{n} c_{j} z_{j} .
$$

We observe that, by (4),

$$
\operatorname{Re} r(z)>1 / 4
$$

whenever $z \in \Psi(X)$. It follows easily from (6) and the Tietze extension theorem that $r$ has an extension $r^{*}$ to all of $T^{n+1}$ which satisfies

$$
\operatorname{Re} r^{*}(z)>1 / 4
$$


for all $z$ in $T^{n+1}$. In particular, $r^{*}$ never vanishes on $T^{n+1}$. Thus $r^{*} / \bar{r}^{*} \in U\left(T^{n+1}\right)$. Using the main result of [5], we can find $F_{1}, F_{2} \in$ $I\left(A\left(T^{n+1}\right)\right)$ such that

$$
\left\|r^{*} / \bar{r}^{*}-F_{1} \bar{F}_{2}\right\|<\varepsilon,
$$

where the norm indicated is of course the sup-norm over $T^{n+1}$. Now (5) can be re-written

$$
\left\|u-r^{*} \circ \Psi / \bar{r}^{*} \circ \Psi\right\|<8 \varepsilon .
$$

Thus, using (7) and (8), we have

$$
\left\|u-F_{1} \circ \Psi \overline{F_{2} \circ \Psi}\right\|<9 \varepsilon .
$$

By Proposition 2 it follows that $F_{1} \circ \Psi$ and $F_{2} \circ \Psi$ belong to $I(A)$. The proof of Theorem 3 is now complete.

Applications and examples. (I) Let $d \mu$ be a measure on some set and let $H^{\infty}(d \mu)$ be a sub-algebra of $L^{\infty}(d \mu)$ which is closed in the essential-sup norm and contains the functions which are constant a.e. We will call a function $h \in H^{\infty}(d \mu)$ inner if $|h|=1$ a.e. The Gelfand transform $f \rightarrow \hat{f}$ is an isometric isomorphism from $L^{\infty}(d \mu)$ onto $C\left(M_{\mu}\right)$, where $M_{\mu}$ denotes the maximal ideal space of $L^{\infty}(d \mu)$. The algebra $H^{\infty}(d \mu)$ is carried by the Gelfand transform onto a closed sub-algebra $\left.\hat{H}^{\infty}\right)(d \mu)$. Furthermore, $h$ is inner in $H^{\infty}(d \mu)$ iff $\hat{h} \in \hat{I}\left(H^{\infty}(d \mu)\right.$. For our purposes it is important to note that every $u \in L^{\infty}(d \mu)$, such that $|u|=1$ a.e., can be written in the form $u=e^{\imath r}$, where $r \in L^{\infty}(d \mu)$. It follows that $U\left(M_{\mu}\right) / \log U\left(M_{\mu}\right)$ is trivial.

By the remarks above and by Theorem 2 we have the following:

COROllaRy 4. Every member of $L^{\infty}(d \mu)$ which is unimodular a.e. can be approximated in the essential sup-norm by quotients of inner functions from $H^{\infty}(d \mu)$ iff $I\left(\hat{H}^{\infty}(d \mu)\right.$ separates the points of $M_{\mu}$.

In [1] Bernard el al show that if $H^{\infty}(d \mu)$ is strongly logmodular, then $I\left(\hat{H}^{\infty}(d \mu)\right)$ separates the points of $M_{\mu}$. Strong logmodularity holds in the classical case where $\mu$ is arc-length measure $\sigma$ on $T^{1}$ and $H^{\infty}(d \sigma)$ is the usual Hardy space. Thus, Corollary 4 and the fact that every inner function in $H^{\infty}(d \mu)$ can be approximated by Blaschke products yield Theorem 1. (See [4, Chapter 10] for details.)

Next consider a plane domain $\Omega$ bounded by a finite number of analytic Jordan curves. Let $H^{\infty}(\Omega)$ denote the algebra of bounded analytic functions on $\Omega$. by Fatou's theorem $H^{\infty}(\Omega)$ can be identified with 
the algebra $H^{\infty}(d s)$ of functions which are non-tangential limits $d s$ - a.e. of functions in $H^{\infty}(\Omega)$. Here of course $d s$ denotes arc length measure on the boundary of $\Omega$. It is shown in [1, Lemma 4.8] that the inner functions in $\hat{H}^{\infty}(d s)$ separate the points of the maximal ideal space of $L^{\infty}(d s)$. Thus, Corollary 4 can be applied to $H^{\infty}(d s)$.

(II) Another application of Theorem 3 occurs in the theory of compact abelian groups. In the discussion which follows $G$ will denote a compact abelian group and $G$ \#ill denote its dual group, i.e., the group of continuous characters on $G$.

We will need the following.

LEMMA 5. Let $S \subseteq G^{\#}$ and let $\langle S\rangle$ denote the subgroup of $G^{\#}$ generated by $S$. If $S$ separates the points of $G$, then $\langle S\rangle=G^{\#}$.

Proof. Suppose that $S$ separates the points of $G$ and that $\langle S\rangle$ is a proper sub-group of $G^{\#}$. Since $G^{\#}$ is discrete, it follows that there is a non-constant character $q$ on $G^{\#}$ such that $q(c)=1$ for all $c \in\langle S\rangle$ (see [7, pp. 35-36]). By the Pontryagin duality theorem there is a point $g_{0} \in G$ such that $q(b)=b\left(g_{0}\right)$ for all $b \in G^{\#}$. In particular, $c\left(g_{0}\right)=1$ for every $c \in S$. Since $S$ separates points it follows that $g_{0}$ must be the identity in $G$. But then $q$ cannot be non-constant, since $q(b)=b\left(g_{0}\right)=1$ for every $b \in G^{\#}$.

THEOREM 6. Suppose that $G$ is either totally disconnected or connected. Let $S$ be a point separating subset of $G^{\#}$ and let $A_{S}$ denote the closed sub-algebra of $C(G)$ generated by $S$. Then $Q\left(A_{S}\right)$ is dense in $U(G)$.

Proof. Suppose $\mathrm{G}$ is totally disconnected. Then $U(G) / \log U(G)$ is trivial. Thus, the hypotheses of Theorem 3 are satisfied by $A_{S}$. It follows that $Q\left(A_{S}\right)$ is dense in $U(G)$.

In the case where $G$ is connected we bring in a result due to Taylor [8, p. 80] which asserts that for each $u \in U(G)$ there is a continuous character $c$ such that $c u \in \log U(G)$. By Lemma 5 it follows that $c \in$ $Q\left(A_{S}\right)$. Thus, condition (i) of Theorem 3 is satisfied by the algebra $A_{S}$. Since $S \subseteq A_{S}$, the condition (ii) is also satisfied. Hence, $Q\left(A_{S}\right)$ is dense in $U(G)$.

(III) It is not hard to give examples where condition (ii) of Theorem 3 fails. If $X$ is a compact subset of the plane and $R(X)$ is the closure of the rational functions in $C(X)$, then the inner functions in $R(X)$ separate the points of $X$ iff $R(X)=C(X)$. A more striking example is provided by an 
algebra constructed by Browder and Wermer in [2, Corollary 1]. The Browder and Wermer algebra is of the form

$$
A(\Psi)=\left\{f \mid f, f \circ \Psi \in A\left(T^{1}\right)\right\},
$$

where $\Psi$ is a homeomorphism of $T^{1}$ onto itself which is singular i.e. $\Psi$ maps a set of Lebesgue measure 0 onto a set of full Lebesgue measure. The algebra $A(\Psi)$ is a Dirichlet sub-algebra of $A\left(T^{1}\right)$. If $h \in I(A(\Psi))$, then both $h$ and $h \circ \Psi$ must be finite Blaschke products. It follows easily that $h$ must be a constant.

Final Remark. Condition (i) of Theorem 2 remains something of a mystery to us. We have been unable to find an example of an algebra of functions which satisfies (ii) but not (i).

Acknowledgments. Part of the work of writing this paper was done while we were on sabbatical leave from Arizona State University during the Spring 1984 semester.

Conversations which we had with Domingo Herrero and David Styer helped us to improve the quality of our results.

We wish, also, to thank the referee for many valuable suggestions and, particularly, for pointing out an error in the original manuscript.

\section{REFERENCES}

[1] A. Bernard, J. B. Garnett and D. E. Marshall, Algebra generated by inner functions, J. Funct. Anal., 25 (1977), 275-285.

[2] A. Browder and J. Wermer, $A$ method for constructing Dirichlet algebras, Proc. Amer. Math. Soc., 15 (1964), 546-552.

[3] R. G. Douglas and W. Rudin, Approximation by inner functions, Pacific J. Math., 31 (1969), 313-320.

[4] K. Hoffman, Banach Spaces of Analytic Functions, Prentice-Hall 1962.

[5] J. N. Mc Donald, Approximation by quotients of rational inner functions, Proc. Amer. Math. Soc., 74, No. 2, (1979), 299-300.

[6] W. Rudin, Function Theory on Polydisks, W. A. Benjamin 1969.

[7] _ Fourier Analysis on Groups, Interscience, 1960.

[8] J. L. Taylor Measure Algebras, C.B.M.S. Regional Conference series \#16.

Received September 10, 1984 and in revised form March 20, 1985.

Arizona State University

TEMPE, AZ 85287 


\section{PACIFIC JOURNAL OF MATHEMATICS EDITORS}

\author{
V. S. VARAdarajan (Managing Editor) \\ University of California \\ Los Angeles, CA 90024 \\ Hebert Clemens \\ University of Utah \\ Salt Lake City, UT 84112 \\ Charles R. DePrima \\ California Institute of Technology \\ Pasadena, CA 91125
}

R. FINN

Stanford University

Stanford, CA 94305

HeRManN FLASChKa

University of Arizona

Tucson, AZ 85721

RAMESH A. GANGOlli

University of Washington

Seattle, WA 98195

ROBION KIRBY

University of California

Berkeley, CA 94720

\author{
C. C. MOORE \\ University of California \\ Berkeley, CA 94720 \\ H. SAMELSON \\ Stanford University \\ Stanford, CA 94305 \\ HAROLD STARK \\ University of California, San Diego \\ La Jolla, CA 92093
}

\section{ASSOCIATE EDITORS}

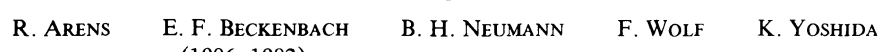

(1906-1982)

\section{SUPPORTING INSTITUTIONS}

\begin{abstract}
UNIVERSITY OF ARIZONA
UNIVERSITY OF BRITISH COLUMBIA

CALIFORNIA INSTITUTE OF TECHNOLOGY

UNIVERSITY OF CALIFORNIA

MONTANA STATE UNIVERSITY

UNIVERSITY OF NEVADA, RENO

NEW MEXICO STATE UNIVERSITY

OREGON STATE UNIVERSITY
\end{abstract}

\author{
UNIVERSITY OF OREGON \\ UNIVERSITY OF SOUTHERN CALIFORNIA \\ STANFORD UNIVERSITY \\ UNIVERSITY OF HAWAII \\ UNIVERSITY OF TOKYO \\ UNIVERSITY OF UTAH \\ WASHINGTON STATE UNIVERSITY \\ UNIVERSITY OF WASHINGTON
}

The Supporting Institutions listed above contribute to the cost of publication of this Journal, but they are not owners or publishers and have no responsibility for its content or policies.

Mathematical papers intended for publication in the Pacific Journal of Mathematics should be in typed form or offset-reproduced (not dittoed), double spaced with large margins. Please do not use built up fractions in the text of the manuscript. However, you may use them in the displayed equations. Underline Greek letters in red, German in green, and script in blue. The first paragraph must be capable of being used separately as a synopsis of the entire paper. In particular it should contain no bibliographic references. Please propose a heading for the odd numbered pages of less than 35 characters. Manuscripts, in triplicate, may be sent to any one of the editors. Please classify according to the scheme of Math. Reviews, Index to Vol. 39. Supply name and address of author to whom proofs should be sent. All other communications should be addressed to the managing editor, or Elaine Barth, University of California, Los Angeles, California 90024.

There are page-charges associated with articles appearing in the Pacific Journal of Mathematics. These charges are expected to be paid by the author's University, Government Agency or Company. If the author or authors do not have access to such Institutional support these charges are waived. Single authors will receive 50 free reprints; joint authors will receive a total of 100 free reprints. Additional copies may be obtained at cost in multiples of 50 .

The Pacific Journal of Mathematics is issued monthly as of January 1966. Regular subscription rate: $\$ 190.00$ a year (5 Vols., 10 issues). Special rate: $\$ 95.00$ a year to individual members of supporting institutions.

Subscriptions, orders for numbers issued in the last three calendar years, and changes of address should be sent to Pacific Journal of Mathematics, P.O. Box 969, Carmel Valley, CA 93924, U.S.A. Old back numbers obtainable from Kraus Periodicals Co., Route 100, Millwood, NY 10546.

The Pacific Journal of Mathematics at P.O. Box 969, Carmel Valley, CA 93924 (ISSN 0030-8730) publishes 5 volumes per year. Application to mail at Second-class postage rates is pending at Carmel Valley, California, and additional mailing offices. Postmaster: Send address changes to Pacific Journal of Mathematics, P.O. Box 969, Carmel Valley, CA 93924.

PUBLISHED BY PACIFIC JOURNAL OF MATHEMATICS, A NON-PROFIT CORPORATION

Copyright $\odot 1986$ by Pacific Journal of Mathematics 


\section{Pacific Journal of Mathematics}

\section{Vol. 122, No. 2 \\ February, 1986}

Gideon Amit and David Chillag, On a question of Feit concerning character values of finite solvable groups ......................257

Constantin Gelu Apostol and Frank Larkin Gilfeather, Isomorphisms modulo the compact operators of nest algebras ................263

Parviz Azimi and James Neil Hagler, Examples of hereditarily $l^{1}$ Banach

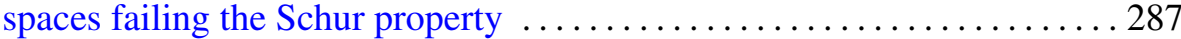

Brian Evan Blank, Boundary behavior of limits of discrete series representations of real rank one semisimple groups . . . . . . . . . . 299

Jeffrey Carroll, Some undecidability results for lattices in recursion theory

Gerald Howard Cliff and Alfred Rheinhold Weiss, Crossed product and

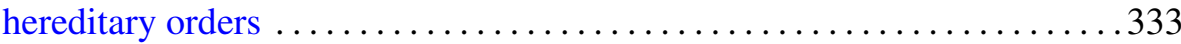

Ralph Cohen, Realizing transfer maps for ramified coverings . . . . . . . . 347

Ronald James Evans, Hermite character sums . .................. 357

C. L. Frenzen and Roderick Sue-Chuen Wong, Asymptotic expansions of the Lebesgue constants for Jacobi series . . . . . . . . . . . . . . . . 391

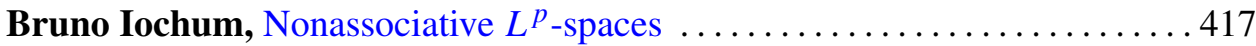

John McDonald, Unimodular approximation in function algebras ....... 435

John Robert Quine, Jr., Ramification and unintegrated value distribution . . 441

Marc Raphael, Commutants of quasisimilar subnormal operators ........ 449

Parameswaran Sankaran and Peter Zvengrowski, On stable

parallelizability of flag manifolds

Helga Schirmer, A relative Nielsen number

Barry Simon, Schrödinger semigroups on the scale of Sobolev spaces . . . . . 475

Viakalathur Shankar Sunder, Stochastic integration in Fock space

Jan de Vries, A note on the $G$-space version of Glicksberg's theorem 